\title{
Corrigendum to "The Effect of PEI and PVP-Stabilized Gold Nanoparticles on Equine Platelets Activation: Potential Application in Equine Regenerative Medicine"
}

\author{
Mateusz Hecold, ${ }^{1}$ Roma Buczkowska, ${ }^{1}$ Aleksandra Mucha, ${ }^{2}$ Jakub Grzesiak, ${ }^{3}$ \\ Olga Rac-Rumijowska, ${ }^{4}$ Helena Teterycz, ${ }^{4}$ and Krzysztof Marycz $^{2,3}$ \\ ${ }^{1}$ Warsaw University of Life Sciences-SGGW, Nowoursynowska 100, 02-797 Warszawa, Poland \\ ${ }^{2}$ Wroclaw University of Environmental and Life Sciences, Kozuchowska 5b, 51-631 Wroclaw, Poland \\ ${ }^{3}$ Wroclaw Research Centre EIT+, Stablowicka 147, 54-066 Wroclaw, Poland \\ ${ }^{4}$ Wroclaw University of Science and Technology, Wybrzeze Wyspianskiego 27, 50-370 Wroclaw, Poland \\ Correspondence should be addressed to Krzysztof Marycz; krzysztofmarycz@interia.pl \\ Received 23 March 2017; Accepted 9 April 2017; Published 11 May 2017
}

Copyright (c) 2017 Mateusz Hecold et al. This is an open access article distributed under the Creative Commons Attribution License, which permits unrestricted use, distribution, and reproduction in any medium, provided the original work is properly cited.

In the article titled "The Effect of PEI and PVP-Stabilized Gold Nanoparticles on Equine Platelets Activation: Potential Application in Equine Regenerative Medicine" [1], an acknowledgment should be added as follows.

\section{Acknowledgments}

This study is conducted by a financial support from Funds for Science at the Faculty of Microsystem Electronics and Photonics, Wroclaw University of Science and Technology (Statutory Activity). Additionally, the article's publication is financially supported by Wroclaw Centre of Biotechnology, programme The Leading National Research Centre (KNOW) for years 2014-2018.

\section{References}

[1] M. Hecold, R. Buczkowska, A. Mucha, J. Grzesiak, and et al., "The effect of pei and pvp-stabilized gold nanoparticles on equine platelets activation: potential application in equine regenerative medicine," Journal of Nanomaterials, vol. 2017, pp. 1-11, 2017. 

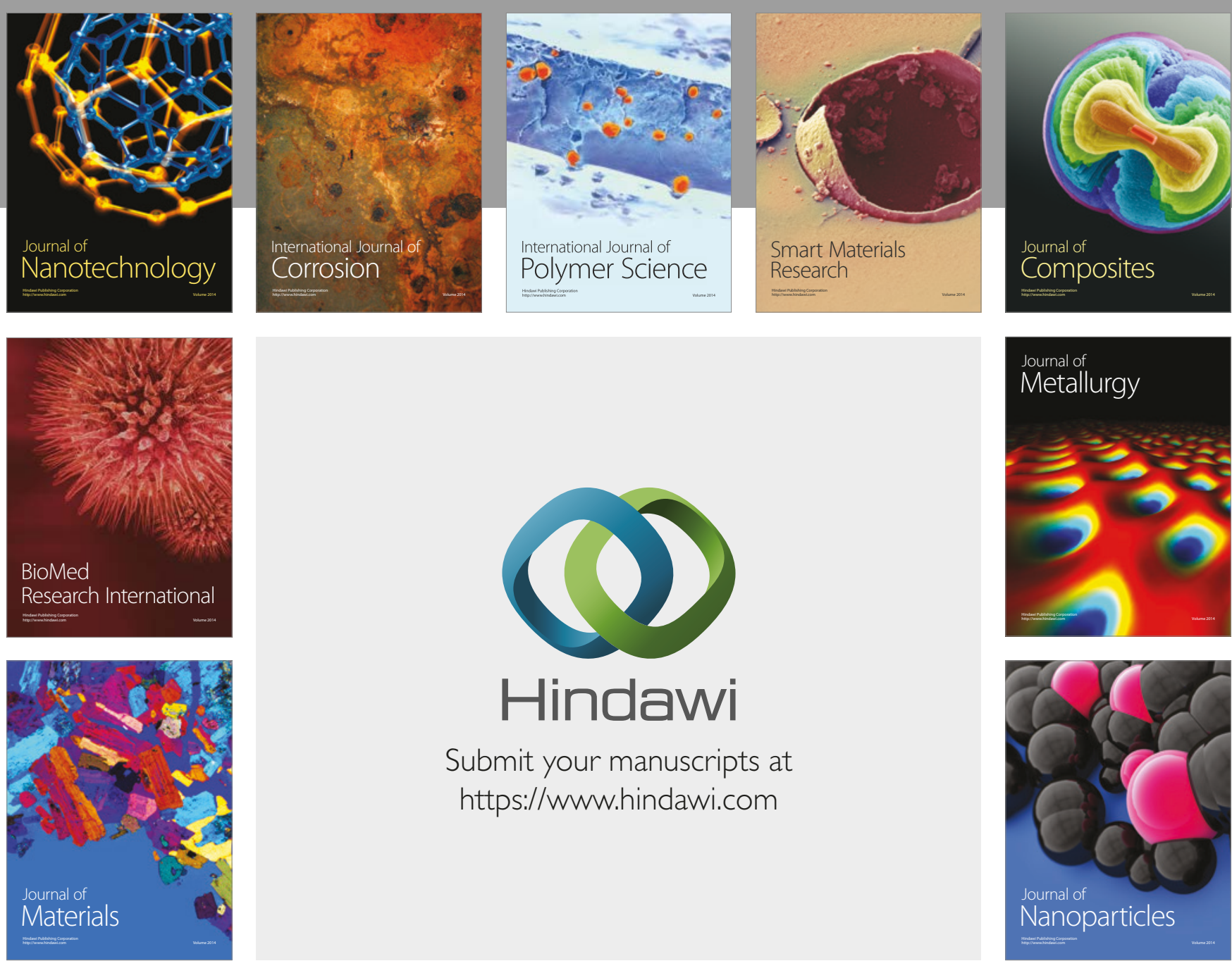

\section{Hindawi}

Submit your manuscripts at

https://www.hindawi.com
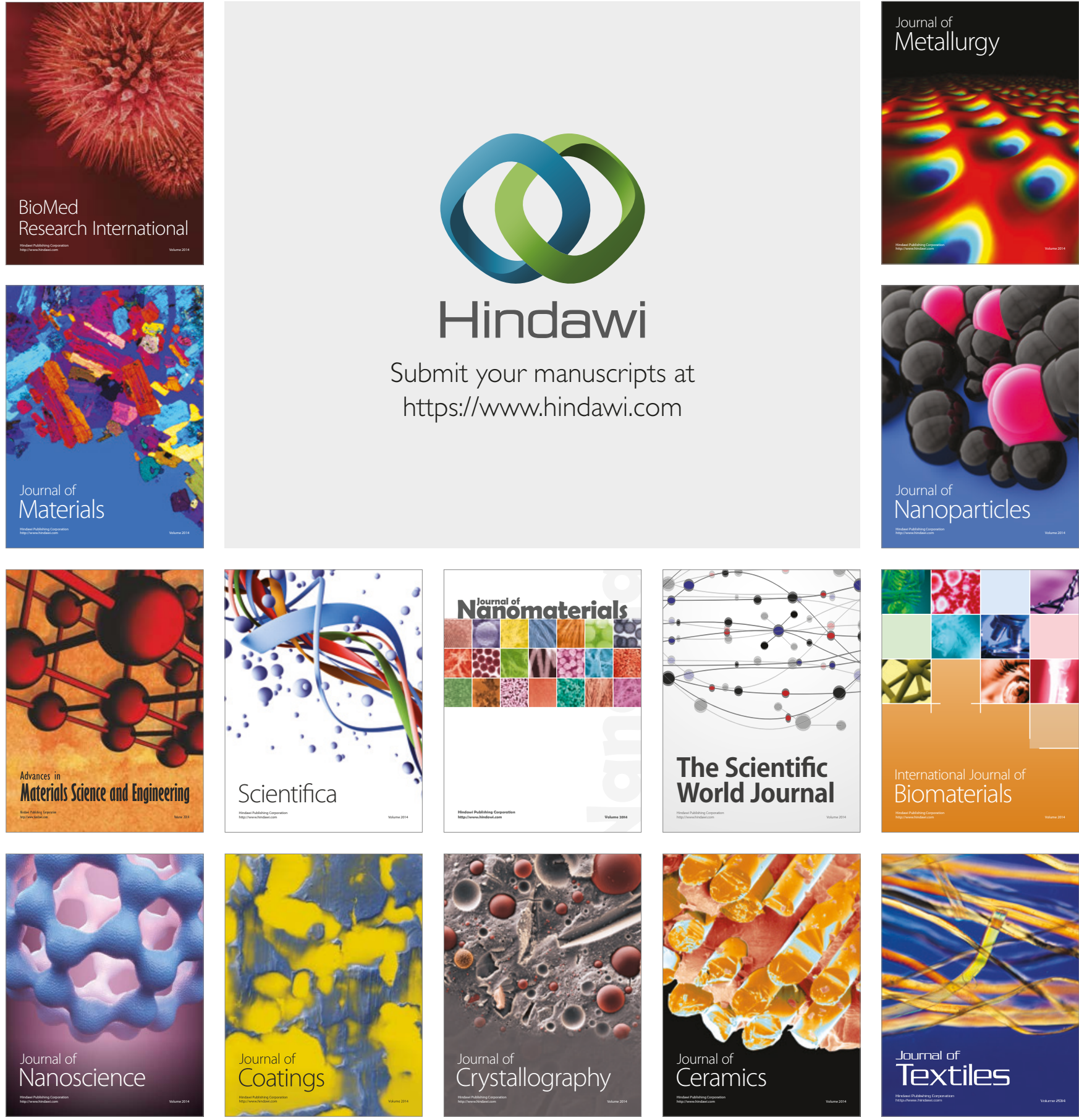

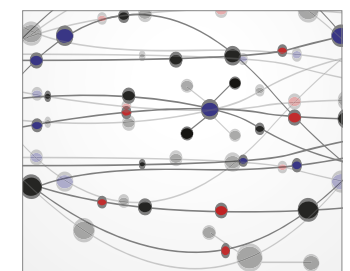

The Scientific World Journal
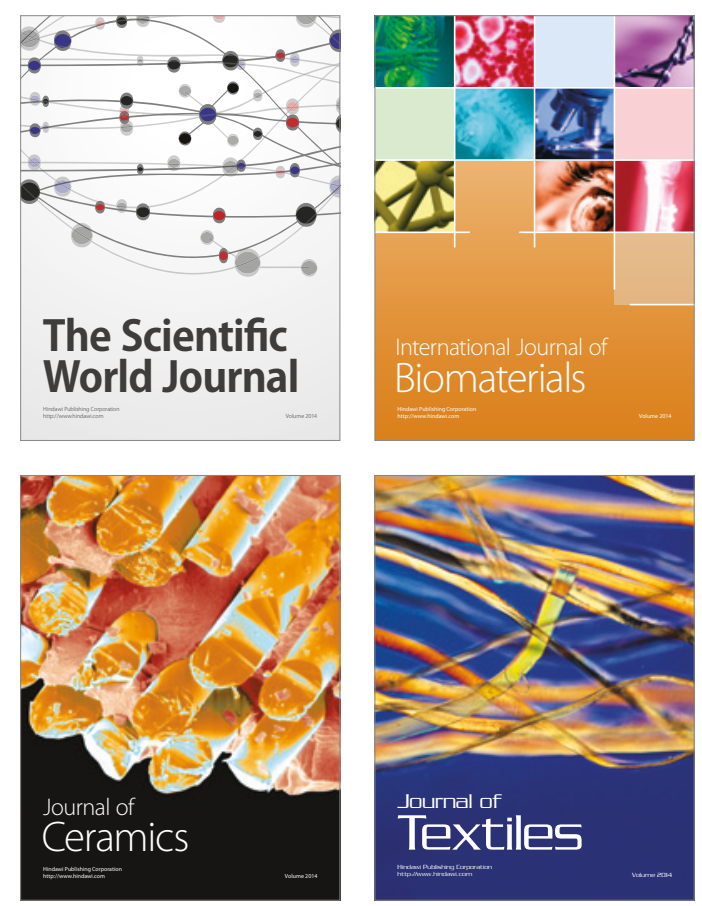